\title{
Shape and spatial correlation control of InAs-InAIAs-InP (001) nanostructure superlattices
}

\author{
W. Lei, Y. H. Chen, ${ }^{\text {a) }}$ P. Jin, X. L. Ye, Y. L. Wang, B. Xu, and Z. G. Wang \\ Key Laboratory of Semiconductor Materials Science, Institute of Semiconductors, \\ Chinese Academy of Sciences, P.O. Box 912, Beijing 100083, People's Republic of China
}

(Received 1 October 2005; accepted 29 December 2005; published online 8 February 2006)

\begin{abstract}
The control of shape and spatial correlation of InAs-InAlAs-InP(001) nanostructure superlattices has been realized by changing the As overpressure during the molecular-beam epitaxy (MBE) growth of InAs layers. InAs quantum wires (QWRs) are obtained under higher As overpressure $\left(1 \times 10^{-5}\right.$ Torr), while elongated InAs quantum dots (QDs) are formed under lower As overpressure $\left(5 \times 10^{-6}\right.$ or $2.5 \times 10^{-6}$ Torr). Correspondingly, spatial correlation changes from vertical anti-correlation in QWR superlattices to vertical correlation in QD superlattices, which is well explained by the different alloy phase separation in InAlAs spacer layers triggered by the InAs nanostrcutures. It was observed that the alloy phase separation in QD superlattices could extend a long distance along the growth direction, indicating the vertical correlation of QD superlattices can be kept in a wide range of spacer layer thickness. (c) 2006 American Institute of Physics.
\end{abstract}

[DOI: $10.1063 / 1.2172288$ ]

Recently, much attention has been devoted to selfassembled InP-based InAs nanostructures due to potential applications in long wavelength $(1.55 \mu \mathrm{m})$ semiconductor lasers and photodetectors. ${ }^{1-4}$ For these applications, it is important to control the shape, size, and distribution of nanostructures, which presents a challenge due to the random nature of the formation of nanostructures. Especially for the InAs-InAlAs-InP (001) system, the control of islands shape is even difficult due to the phase separation effect in InAlAs layers ${ }^{5,6}$ and the relatively weak driving force for the islands formation associated with low misfit strain (3.2\%). Though InAs-InAlAs quantum wire (QWR) superlattices have been grown by several research groups, ${ }^{6-9}$ the fabrication of InAsInAlAs quantum dot (QD) superlattices has not been reported so far.

During the growth of nanostructure superlattices, the buried nanostructures tend to influence nucleation sites in the subsequent layer, leading to the formation of vertically and laterally ordered nanostructure superlattices. Depending on material systems, various types of spatial correlation have been reported, ranging from vertical correlation in $\mathrm{Ge}-\mathrm{Si}$ and InAs-GaAs nanostructure superlattices to face-center-cubic (fcc) stacking sequences in $\mathrm{PbSe}-\mathrm{PbEuTe}$ nanostructure superlattices. ${ }^{10,11}$ These different stacking sequences may have great potential for realizing some concepts of quantum devices. For InAs-InAlAs nanostructure superlattices, the vertical anti-correlation in InAs-InAlAs QWR superlattices has been reported in previous works. ${ }^{6-8}$ However, no vertical correlation of these InAs-InAlAs nanostructure superlattices is observed despite the effort of several research groups. ${ }^{6-8}$

In this work, we demonstrate the control of shape and spatial correlation of InAs-InAlAs-InP(001) nanostructure superlattices by changing As overpressure during the growth of InAs layers. It is shown that InAs QWRs or elongated QDs can be obtained through varying As overpressure during the growth of InAs layers. Correspondingly, the spatial correlation changes from vertical anti-correlation in QWR su-

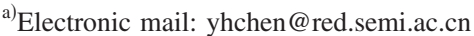

perlattices to vertical correlation in QD superlattices. The important role of the alloy phase separation in InAlAs spacer layers has been discussed.

The samples were grown on semi-insulating InP (001) substrates with an $\mathrm{As}_{2}$ source using a solid source molecular beam epitaxy system (Riber 32P). The samples were composed of a $200 \mathrm{~nm}-\mathrm{In}_{0.52} \mathrm{Al}_{0.48} \mathrm{As}$ buffer layer, six periods of $3 \mathrm{ML}-\mathrm{InAs} / 15 \mathrm{~nm}-\mathrm{In}_{0.52} \mathrm{Al}_{0.48} \mathrm{As}$ and an $80 \mathrm{~nm}$ $-\mathrm{In}_{0.52} \mathrm{Al}_{0.48} \mathrm{As}$ cap layer. The InAlAs layers were grown at the temperature of $510{ }^{\circ} \mathrm{C}$ under an As overpressure of $5 \times 10^{-6}$ Torr. Before the InAs deposition, the growth was interrupted for about 2.5 min under a $5 \times 10^{-6}$ Torr As overpressure. The InAs layers were grown at $500{ }^{\circ} \mathrm{C}$ under As overpressures of $1 \times 10^{-5}$ Torr, $5 \times 10^{-6}$ Torr, and 2.5 $\times 10^{-6}$ Torr for samples A-C, respectively. After the InAs deposition, a growth interruption of $30 \mathrm{~s}$ was introduced, and then the As overpressure was tuned to $5 \times 10^{-6}$ Torr and InAlAs was deposited. The growth rate of InAlAs and InAs layers were $0.86 \mu \mathrm{m} / \mathrm{h}$ and $0.42 \mathrm{ML} / \mathrm{s}$. The InAs surface reconstruction was observed by reflection high energy electron diffraction (RHEED). The structural properties of the samples were studied by transmission electron microscopy (TEM) measurements.

The $g=002$ cross-sectional dark-field (DF) TEM images of the samples are shown in Fig. 1. The shape of the InAs islands are quite different depending on the As overpressure during the growth of InAs layers. For the InAs layers grown under $1 \times 10^{-5}$ Torr As overpressure, InAs QWRs oriented along the [1-10] direction are formed. When the As overpressure is decreased to $5 \times 10^{-6}$ or $2.5 \times 10^{-6}$ Torr, InAs QDs elongated along the [1-10] direction are obtained. The structure parameters of the islands in the three samples are listed in Table I. Clearly, as the As overpressure is decreased, the size of InAs nanostructures along the [1-10] direction is decreased from $\sim 200$ to $\sim 25 \mathrm{~nm}$, while their [110]-oriented sizes are around 16-18 $\mathrm{nm}$. The As overpressure has little effect on the base width and lateral periodicity along the [110] direction of the InAs islands here. This is in contrast to the InAs-GaAs system, where As overpressure exhibited sig- 

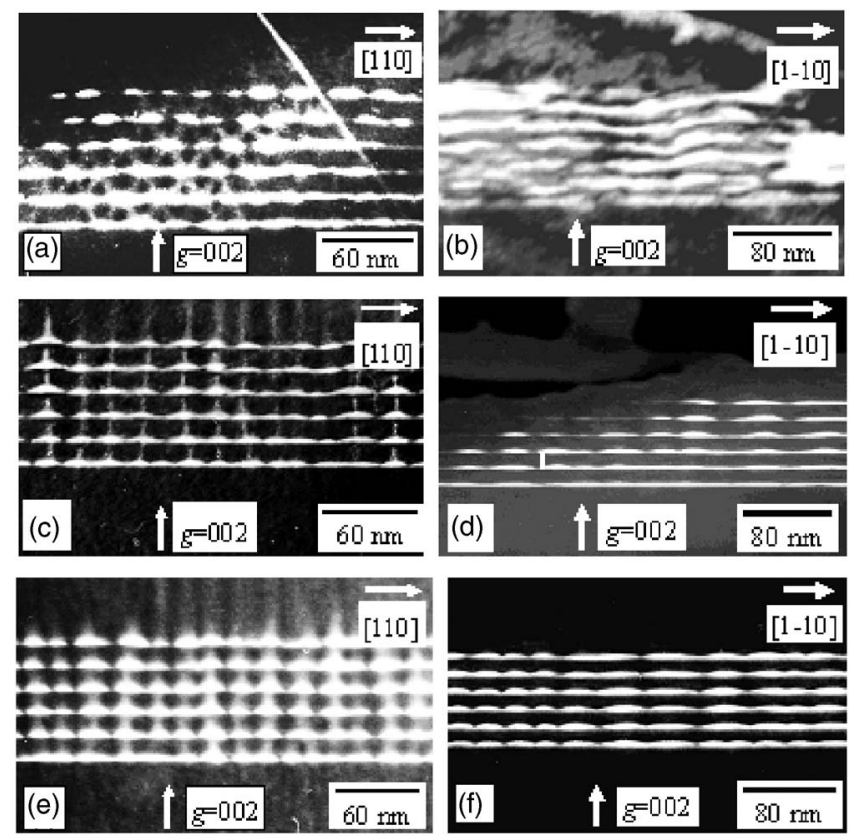

FIG. 1. $g=002$ [1-10] and [110] cross-sectional dark field TEM images of the samples. (a) and (b) for sample A; (c) and (d) for sample B; (e) and (f) for sample $\mathrm{C}$.

nificant influence on the size and density of the islands. ${ }^{12}$ The similar insensitivity that varying growth rate and deposition thickness has little effect on the [110]-oriented size and density of the InAs-InAlAs QWRs was previously reported, which was attributed to the lateral composition modulation (LCM) formed in the InAlAs buffer layer. ${ }^{5}$ The effect of the LCM in InAlAs on the formation of the InAs QWRs was clearly evidenced by the TEM study on InAs-InAlAs QWRs grown on $\operatorname{InP}(001)$ misoriented substrate. ${ }^{13}$

The different shapes of InAs islands in the samples can be mainly ascribed to the different InAs surface reconstructions observed by RHEED: $(2 \times 4)$ under $1 \times 10^{-5}$ Torr As overpressure or $(2 \times 1)$ under $2.5 \times 10^{-6}$ and $5 \times 10^{-6}$ Torr As overpressure. As suggested by previous works, ${ }^{7,14}$ the Asstabilized InAlAs surface will present an anisotropic surface roughness, which can be modified by varying the As coverage on the surface. For sample A under a higher As coverage, the $(2 \times 4)$ surface reconstruction will induce considerable surface anisotropy in the InAlAs layers, leading to the formation of QWRs. When the As overpressure is decreased to $5 \times 10^{-6}$ or $2.5 \times 10^{-6}$ Torr, this anisotropic surface roughness will become less obvious due to the $(2 \times 1)$ surface reconstruction. This will favor the formation of more isotropic islands (QDs).

As shown in Fig. 1, quite different spatial correlations of the InAs nanostructure superlattices are observed for the samples. For sample A, the InAs-InAlAs QWR superlattices

TABLE I. Height $(H)$, base length and lateral periodicity along the [110] direction $\left(L_{110}\right.$ and $\left.\lambda_{110}\right)$ and base length along the [1-10] direction $\left(L_{1-10}\right)$ of the InAs islands in the samples.

\begin{tabular}{clccc}
\hline \hline Sample & \multicolumn{1}{c}{$H(\mathrm{~nm})$} & $L_{110}(\mathrm{~nm})$ & $L_{1-10}(\mathrm{~nm})$ & $\lambda_{110}(\mathrm{~nm})$ \\
\hline A & $6.5 \pm 0.7$ & $18 \pm 3$ & $190 \pm 60$ & $23 \pm 5$ \\
B & $6.7 \pm 1$ & $18 \pm 4$ & $34 \pm 7$ & $21 \pm 2$ \\
C & $6.1 \pm 1.2$ & $16 \pm 3$ & $25 \pm 7$ & $19 \pm 3$ \\
\hline \hline
\end{tabular}

show strong vertical anti-correlation, i.e., the QWRs of upper layer are centered in the interstices of QWRs of the previous layer. As to samples B and C, the InAs-InAlAs QD superlattices exhibit vertical correlation, i.e., the QDs of upper layer are located just above QDs of the previous layer.

Previous works based on elastic properties (anisotropic/ isotropic) of the matrix material predict that spatial correlation of QWR superlattices can be turned from vertical anticorrelation to vertical correlation by changing spacer layer thickness or island size. ${ }^{11,15}$ However, the vertical correlation in InAs-InAlAs QWR superlattices is not observed experimentally despite the effort of $\mathrm{Li}$ et al. and Brault et al ${ }^{6,7} \mathrm{It}$ was suggested that spatial correlation of InAs-InAlAs nanostructure superlattices is determined not by the elastic properties of matrix, but by the alloy decomposition in spacer layers. ${ }^{6,8,16}$ The TEM results shown in Fig. 1 confirm the close relation between the alloy phase separation and the spatial correlation of nanostructures. From Figs. 1(a), 1(c), and 1(e), one can observe the fine alternate bright/dark contrast regions in the InAlAs spacer layers. Because 002 diffraction is much more sensitive to chemical composition than to strain, the contrast observed in the $g=002$ crosssectional TEM images should be related to composition variation. Therefore, the fine alternate bright/dark contrast regions reveal a LCM along the [110] direction, where the brighter regions are In-rich and the darker regions $\mathrm{Al}$ rich. For sample A, the LCM in InAlAs spacer layers forms V-like In-rich InAlAs arms above the QWRs, which is also observed in previous researches. ${ }^{6,8}$ The meeting points of these V-like In-rich arms will provide adequate nucleation sites for new InAs QWRs in the next plane of stacked structure taking into account the smaller misfit strain energy. ${ }^{6,8,17}$ leading to the vertical anti-correlation of QWR superlattices. However, as to samples B and C, the alloy phase separation in InAlAs spacer layers forms I-like In-rich arms just above the QDs. These I-like In-rich arms originate from each InAs QD and align along the [001] direction, as shown in Figs. 1(c) and 1(e). Similar to the V-like In-rich arms in InAlAs spacer layers of sample A, these I-like In-rich arms will also provide preferential nucleation sites for new InAs QDs in the subsequent plane of the stacked structure, resulting in the vertical spatial correlation.

In addition, it is observed that the alloy phase separation triggered by the InAs QDs can extend to the sample surface, which is $80 \mathrm{~nm}$ away from the topmost InAs layer. Figure 2 shows the $g=002[1-10]$ cross-sectional TEM images of typical In-rich regions in InAlAs cap layer of sample C. This indicates that the vertical correlation of QD superlattices can be kept when spacer-layer is thinner than $80 \mathrm{~nm}$. ${ }^{6}$ Though In-rich regions in InAlAs cap layer of the QWR superlattices cannot be observed clearly for sample A, our previous work on InAs-InAlAs QWR superlattices shows that the V-like In-rich region can extend at least $35 \mathrm{~nm}$ along the growth direction.

The exact explanation of the alloy phase separation in InAlAs spacer layers induced by buried InAs QDs and QWRs is still lacking. It has been reported that when burying strained InAs islands with immiscible InAlAs alloy, the starting surface morphology and inhomogeneous surface strain have much influence on the phase separation in the alloy. ${ }^{19-21}$ A comprehensive simulation of the phase separation in InAlAs spacer layers should take into account the material parameters, islands shape, surface strain distribution, detailed Downloaded 22 Feb 2010 to 150.203.243.41. Redistribution subject to AlP license or copyright; see http://apl.aip.org/apl/copyright.jsp 


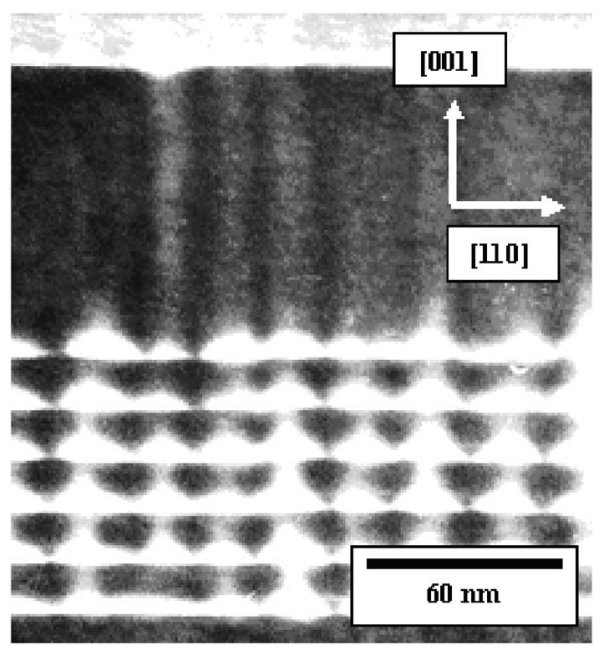

FIG. 2. $g=002$ [1-10] cross-sectional dark-field TEM images of typical In-rich regions in InAlAs cap layer of sample C. The image contrast has been enhanced to see the In-rich regions more clearly.

structure of the growth front and kinetic effects, which goes beyond the scope of this paper.

In conclusion, the islands shape in InAs-InAlAs$\operatorname{InP}(001)$ nanostructure superlattices is strongly dependent on the As overpressure during the growth of InAs layers. When the As overpressure is decreased from $1 \times 10^{-5}$ Torr to 5 $\times 10^{-6}$ or $2.5 \times 10^{-6}$ Torr, the shape of InAs-InAlAs nanostructure superlattices is turned from QWR superlattices into QD superlattices. Accordingly, the spatial correlation is changed from vertical anti-correlation in QWR superlattices to vertical correlation in QD superlattices. The formation of these different spatial correlations is attributed to the different alloy phase separations in InAlAs spacer layers induced by the buried QWRs or QDs.
This work was supported by Special Funds for Major State Basic Research Project of China (No. G2000068303), National Natural Science Foundation of China (Nos. 60390074, 90201007, 90301007).

${ }^{1}$ J. S. Kim, J. H. Lee, S. U. Hong, W. S. Han, H.-S. Kwack, C. W. Lee, and D. K. Oh, Appl. Phys. Lett. 85, 1033 (2004).

${ }^{2}$ O. Bierwagen and W. T. Masselink, Appl. Phys. Lett. 86, 113110 (2005).

${ }^{3}$ E. Finkman, S. Maimon, V. Immer, G. Bahir, S. E. Schacham, F. Fossard,

F. H. Julien, J. Brault, and M. Gendry, Phys. Rev. B 63, 045323 (2001).

${ }^{4}$ F. Fossard, F. H. Julien, E. Péronne, A. Alexandrou, J. Brault, and M. Gendry, Infrared Phys. Technol. 42, 443 (2001).

${ }^{5}$ F. A. Zhao, Y. H. Chen, X. L. Ye, P. Jin, B. Xu, Z. G. Wang, and

C. L. Zhang, J. Phys.: Condens. Matter 16, 7603 (2004).

${ }^{6}$ J. Brault, M. Gendry, O. Marty, M. Pitaval, J. Olvares, G. Grenet, and G. Hollinger, Appl. Surf. Sci. 162/163, 584 (2000).

${ }^{7}$ H. Li, T. Daniels-Race, and M.-A. Hasan, Appl. Phys. Lett. 80, 1367 (2002).

${ }^{8}$ Z. Sun, S. F. Yoon, J. Wu, and Z. Wang, J. Appl. Phys. 91, 6021 (2002).

${ }^{9}$ W. Lei, Y. H. Chen, B. Xu, X. L. Ye, Y. P. Zeng, and Z. G. Wang, Nanotechnology 16, 1974 (2005).

${ }^{10}$ V. A. Shchukin and D. Bimberg, Rev. Mod. Phys. 71, 1125 (1999).

${ }^{11}$ G. Springholz, M. Pinczolits, P. Mayer, V. Holy, G. Bauer, H. H. Kang, and L. Salamanca-Riba, Phys. Rev. Lett. 84, 4669 (2000).

${ }^{12}$ H. Yasuda, F. Matsukura, Y. Ohno, and H. Ohno, Appl. Surf. Sci. 166, 413 (2000).

${ }^{13}$ Y.-l. Wang, P. Jin, X. L. Ye, C. L Zhang, G. X. Shi, R. Y. Li, Y. H. Chen, and Z. G. Wang (unpublished).

${ }^{14}$ J. Brault, M. Gendry, G. Grenet, G. Hollinger, J. Olivares, B. Salem, T. Benyattou, and G. Bremond, J. Appl. Phys. 92, 506 (2002).

${ }^{15}$ V. A. Shchukin, D. Bimberg, V. G. Malyshkin, and N. N. Ledentsov, Phys. Rev. B 57, 12262 (1998).

${ }^{16}$ C. Priester and G. Grenet, Phys. Rev. B 64, 125312 (2001).

${ }^{17}$ M. Gendry, J. Brault, B. Salem, G. Bremond, and O. Marty, Physica E (Amsterdam) 17, 505 (2003).

${ }^{18}$ Y. L. Wang, Y. H. Chen, J. Wu, Z. G. Wang, and Y. P. Zeng, Superlattices Microstruct. 38, 151 (2005).

${ }^{19}$ J. Tersoff, Phys. Rev. Lett. 77, 2017 (1996).

${ }^{20}$ F. Glas, Phys. Rev. B 55, 11277 (1997).

${ }^{21}$ C. Priester and G. Grenet, Phys. Rev. B 61, 16029 (2000). 